UDC 517.518.86, 517.218.244, 517.927.2

S. I. Butt, B. BAYraktar, M. Umar

\title{
SEVERAL NEW INTEGRAL INEQUALITIES VIA $K$-RIEMANN-LIOUVILLE FRACTIONAL INTEGRALS OPERATORS
}

\begin{abstract}
The main objective of this paper is to establish several new integral inequalities including $k$-Riemann-Liouville fractional integrals for convex, $s$-Godunova-Levin convex functions, quasiconvex, $\eta$-quasi-convex. In order to obtain our results, we have used classical inequalities as Hölder inequality, Power mean inequality and Weighted Hölder inequality. We also give some applications.
\end{abstract}

Key words: $\eta$-quasi-convex, s-Godunova-Levin type, k-Riemann-Liouville fractional integral, Hölder inequality, weighted Hölder inequality, power mean inequality

2010 Mathematical Subject Classification: 26A33, 26A51, $26 D 15$

1. Introdaction. In the recent years, theory of inequalities has been attracting many researchers due to its applications in our daily life and within the mathematics. Theory of convex functions has great importance in various fields of pure and applied sciences. It is known that theory of convex functions is closely related to theory of inequalities. It has now become a trending aspect of mathematical research to generalize classical known results via the fractional integral operator.

Fractional calculus may be described as an extension of the concept of derivative operator from integer order $n$ to arbitrary order $a$. Fractional derivative has been considered as the inverse of a fractional integral. Considerable work has been done in the recent years on fractional integrals because of their applications in many fields of science and technology. The fractional integrals are a powerful tool in applied mathematics, used to solve many problems from different fields of science and engineering.

The Riemann-Liouville integral is named after Bernhard Riemann and Joseph Liouville, the latter was the first to consider the possibility of fractional calculus in 1832 . The concept of $k$-Riemann-Liouville fractional (C) Petrozavodsk State University, 2021 
integral is an important extension of Riemann-Liouville fractional integrals. We want to stress here that for $k \neq 1$, the properties of $k$-RiemannLiouville fractional integrals are quite dissimilar from those of general Riemann-Liouville fractional integrals. For this, the $k$-Riemann-Liouville fractional integrals have aroused interest of many researchers. Our concern is the $k$-Riemann-Liouville fractional integral operator. We invite the interested reader to see [5], [17], [13], [16], [11] and references therein.

The following definitions are well-known:

Definition 1. A function $f:[a, b] \rightarrow \mathbb{R}$ is said to be convex if

$$
f(t x+(1-t) y) \leqslant t f(x)+(1-t) f(y)
$$

for all $x, y \in[a, b]$ and $t \in[0,1]$.

Many important inequalities are established for the class of convex functions; one of the most important is called Hermite-Hadamard's inequality (or Hadamard's inequality). This double inequality is stated as follows:

Let $f: I \subseteq \mathbb{R} \rightarrow \mathbb{R}$ be a convex function and let $a, b \in I$, with $a<b$. Then

$$
f\left(\frac{a+b}{2}\right) \leqslant \frac{1}{b-a} \int_{a}^{b} f(x) d x \leqslant \frac{f(a)+f(b)}{2} .
$$

This inequality is in the reversed direction if $f$ is concave.

Definition 2. [9] The function $f:[a, b] \rightarrow \mathbb{R}$ is said to be $\eta$-convex if the inequality

$$
f(t x+(1-t) y) \leqslant f(y)+t \eta(f(x), f(y))
$$

holds for all $x, y \in[a, b]$ and $t \in[0,1]$, and $\eta$ is defined by

$$
\eta: f([a, b]) \times f([a, b]) \rightarrow \mathbb{R} .
$$

If we set $\eta(x, y)=x-y$ in the definition above, we can directly obtain the classical definition of a convex function.

Definition 3. [9] Let a real function $f$ be defined on some non-empty interval $I$ of real numbers. The function $f$ is said to be quasi-convex on $I$ if following inequality holds:

$$
f(t x+(1-t) y) \leqslant \max \{f(x), f(y)\}
$$


Also, $f$ is called $\eta$-quasi-convex if

$$
f(t x+(1-t) y) \leqslant \max \{f(y), f(y)+\eta(f(x), f(y))\}
$$

holds for all $x, y \in I$ and $t \in[0,1]$.

Clearly, any convex function is a QC function, though not every QC function is convex.

For example the function $f: R^{+} \rightarrow R, f(x)=\ln (x), x \in R^{+}$is quasi-convex but it is not convex.

Definition 4. [8] we say that the function $f: C \subset X \rightarrow[0, \infty)$ is of $s$-Godunova-Levin type, with $s \in[0,1]$, if the inequality

$$
f(t x+(1-t) y) \leqslant t^{-s} f(x)+(1-t)^{-s} f(y) \quad(s-G L)
$$

holds for all $t \in(0,1)$ and $x, y \in C$.

Now we give a necessary definition of fractional calculus theory, which is used throughout this paper.

Definition 5. [14] Let $f \in L_{1}[a, b]$. The $k$-Riemann-Liouville integrals ${ }_{k} J_{a^{+}}^{\alpha} f$ and ${ }_{k} J_{b^{-}}^{\alpha} f$ of order $\alpha>0$ with $a \geqslant 0$ are defined by

$$
{ }_{k} J_{a^{+}}^{\alpha} f(x)=\frac{1}{k \Gamma_{k}(\alpha)} \int_{a}^{x}(x-t)^{\frac{\alpha}{k}-1} f(t) d t, \quad x>a, k>0
$$

and

$$
{ }_{k} J_{b-}^{\alpha} f(x)=\frac{1}{k \Gamma_{k}(\alpha)} \int_{x}^{b}(t-x)^{\frac{\alpha}{k}-1} f(t) d t, \quad x<b, k>0
$$

respectively, where $\Gamma_{k}(\cdot)$ stands for the $k$-Gamma function.

For a positive real number $k$ and a complex number $\alpha$ with $\operatorname{Re}(\alpha)>0$, the $k$-Gamma function is given by the integral [7]:

$$
\Gamma_{k}(\alpha)=\int_{0}^{\infty} t^{\alpha-1} e^{-\frac{t^{k}}{k}} d t \text { with } \alpha \Gamma_{k}(\alpha)=\Gamma_{k}(\alpha+k) .
$$

Remark. If $k=1$, then $k$-Riemann-Liouville fractional integrals reduce to the classical Riemann-Liouville fractional integral. And, if $\alpha=1$ and $k=1$, the fractional integral reduces to the classical integral. 
For some recent results connected with fractional integral inequalities, see [1], [3], [6], [12].

The main aim of this paper is to establish three new integral identities and to prove, using these equalities, some new integral inequalities for $\eta$-QC and $s$-GL convex functions via the $k$-Riemann - Liouville fractional integral operators.

2. Main Results. In order to move further, we need the following useful lemma.

Lemma 1. Let $f: I \subset \mathbb{R} \rightarrow \mathbb{R}$ be a differentiable function on $I^{\circ}$, where $a, b \in I$ with $t \in[0,1]$. If $f^{\prime} \in L[a, b]$, then for all $a \leqslant x<y \leqslant b$ and $k, \alpha>0$, we have:

$$
\frac{f(y)}{y-x}-\frac{\Gamma_{k}(\alpha+k)}{(y-x)^{\frac{\alpha}{k}+1}}{ }_{k} J_{y^{-}}^{\alpha} f(x)=\int_{0}^{1}(1-t)^{\frac{\alpha}{k}} f^{\prime}(t x+(1-t) y) d t .
$$

Proof. Integrate by parts:

$$
\begin{aligned}
\int_{0}^{1}(1-t)^{\frac{\alpha}{k}} f^{\prime}(t x+ & (1-t) y) d t= \\
& =\frac{f(y)}{y-x}-\frac{\frac{\alpha}{k}}{y-x} \int_{0}^{1}(1-t)^{\frac{\alpha}{k}-1} f(t x+(1-t) y) d t .
\end{aligned}
$$

Change the variable $u=t x+(1-t) y$ to these integrals, and get

$$
\begin{aligned}
\frac{1}{y-x} f(y)-\frac{\frac{\alpha}{k}}{(y-x)^{\frac{\alpha}{k}+1}} \int_{x}^{y}(u-x)^{\frac{\alpha}{k}-1} f(u) d u & = \\
& =\frac{1}{y-x} f(y)-\frac{\Gamma_{k}(\alpha+k)}{(y-x)^{\frac{\alpha}{k}+1}}{ }_{k} J_{y^{-}}^{\alpha} f(x) .
\end{aligned}
$$

This completes the proof.

Theorem 1. Let $f: I \subset \mathbb{R} \rightarrow \mathbb{R}, I \subset[0, \infty)$, be a differentiable function on $I^{\circ}$, such that $f^{\prime} \in L[a, b]$, where $a, b \in I$, with $a \leqslant x<y \leqslant b$. If $f^{\prime}$ is $\eta-Q C$ on $[x, y]$ for $t \in[0,1]$, then we have 


$$
\begin{aligned}
& \frac{1}{y-x} f(y)-\frac{\Gamma_{k}(\alpha+k)}{(y-x)^{\frac{\alpha}{k}+1}}{ }_{k} J_{y^{-}}^{\alpha} f(x) \leqslant \\
& \leqslant\left(\frac{k}{\alpha+k}\right) \max \left\{f^{\prime}(y), f^{\prime}(y)+\eta\left(f^{\prime}(x), f^{\prime}(y)\right)\right\}
\end{aligned}
$$

for all $k, \alpha>0$.

Proof. Since $f^{\prime}(t x+(1-t) y) \leqslant \max \left\{f^{\prime}(y), f^{\prime}(y)+\eta\left(f^{\prime}(x), f^{\prime}(y)\right)\right\}$ for $t \in[0,1]$, we obtain, from Lemma 1 :

$$
\begin{gathered}
\frac{1}{y-x} f(y)-\frac{\Gamma_{k}(\alpha+k)}{(y-x)^{\frac{\alpha}{k}}+1} k J_{y^{-}}^{\alpha} f(x)=\int_{0}^{1}(1-t)^{\frac{\alpha}{k}} f^{\prime}(t x+(1-t) y) d t \leqslant \\
\leqslant \max \left\{f^{\prime}(y), f^{\prime}(y)+\eta\left(f^{\prime}(x), f^{\prime}(y)\right)\right\} \int_{0}^{1}(1-t)^{\frac{\alpha}{k}} d t= \\
=\left(\frac{k}{\alpha+k}\right) \max \left\{f^{\prime}(y), f^{\prime}(y)+\eta\left(f^{\prime}(x), f^{\prime}(y)\right)\right\} .
\end{gathered}
$$

This completes the proof.

Corollary 1. If we set $\eta(x, y)=x-y$ in Theorem 1 , we obtain

$$
\frac{1}{y-x} f(y)-\frac{\Gamma_{k}(\alpha+k)}{(y-x)^{\frac{\alpha}{k}+1}} J_{k} J_{y^{-}}^{\alpha} f(x) \leqslant\left(\frac{k}{\alpha+k}\right) \max \left\{f^{\prime}(y), f^{\prime}(x)\right\} .
$$

Corollary 2. For $x=a$ and $y=b$, we obtain

$$
\begin{aligned}
\frac{1}{b-a} f(b)-\frac{\Gamma_{k}(\alpha+k)}{(b-a)^{\frac{\alpha}{k}+1}} J_{b-}^{\alpha} f(a) \leqslant\left(\frac{k}{\alpha+k}\right) \max \left\{f^{\prime}(b), f^{\prime}(a)\right\} \leqslant \\
\leqslant\left(\frac{k}{\alpha+k}\right)\left\|f^{\prime}\right\|_{\infty},
\end{aligned}
$$

where $\left\|f^{\prime}\right\|_{\infty}=\sup \left|f^{\prime}(x)\right|$. Moreover, if we choose $k=\alpha=1$, we obtain

$$
f(b)-\frac{1}{b-a} \int_{a}^{b} f(x) d x \leqslant\left(\frac{b-a}{2}\right) \max \left\{f^{\prime}(b), f^{\prime}(a)\right\} .
$$

Theorem 2. Let $f: I \subset \mathbb{R} \rightarrow \mathbb{R}, I \subset[0, \infty)$, be a differentiable function on $I^{\circ}$, such that $f^{\prime} \in L[a, b]$, where $a, b \in I, a \leqslant x<y \leqslant b$. If $\left|f^{\prime}\right|^{q}$ is $\eta-Q C$ on $[x, y]$ for $t \in[0,1], q>1, p=\frac{q}{q-1}$, then we have 


$$
\begin{aligned}
& \left|\frac{1}{y-x} f(y)-\frac{\Gamma_{k}(\alpha+k)}{(y-x)^{\frac{\alpha}{k}+1}}{ }_{k} J_{y^{-}}^{\alpha} f(x)\right| \leqslant \\
& \quad \leqslant\left(\frac{k}{\alpha p+k}\right)^{\frac{1}{p}} \max \left\{\left|f^{\prime}(y)\right|^{q},\left|f^{\prime}(y)\right|^{q}+\eta\left(\left|f^{\prime}(x)\right|^{q},\left|f^{\prime}(y)\right|^{q}\right)\right\}^{\frac{1}{q}} .
\end{aligned}
$$

for all $k, \alpha>0$.

Proof. First of all, we know that

$$
\left|f^{\prime}(t x+(1-t) y)\right|^{q} \leqslant\left[\max \left\{\left|f^{\prime}(y)\right|^{q},\left|f^{\prime}(y)\right|^{q}+\eta\left(\left|f^{\prime}(x)\right|^{q},\left|f^{\prime}(y)\right|^{q}\right)\right\}\right] .
$$

Using the well known Hölder inequality, properties of the absolute value, and using Lemma 1, we obtain

$$
\begin{gathered}
\left|\frac{1}{y-x} f(y)-\frac{\Gamma_{k}(\alpha+k)}{(y-x)^{\frac{\alpha}{k}+1}}{ }_{k} J_{y^{-}}^{\alpha} f(x)\right|=\left|\int_{0}^{1}(1-t)^{\frac{\alpha}{k}} f^{\prime}(t x+(1-t) y) d t\right| \leqslant \\
\leqslant \int_{0}^{1}\left|(1-t)^{\frac{\alpha}{k}}\right|\left|f^{\prime}(t x+(1-t) y)\right| d t \leqslant \\
\leqslant\left(\int_{0}^{1}(1-t)^{\frac{\alpha p}{k}} d t\right)^{\frac{1}{p}}\left(\left|f^{\prime}(t x+(1-t) y)\right|^{q} d t\right)^{\frac{1}{q}}= \\
=\left(\frac{k}{\alpha p+k}\right)^{\frac{1}{p}}\left[\max \left\{\left|f^{\prime}(y)\right|^{q},\left|f^{\prime}(y)\right|^{q}+\eta\left(\left|f^{\prime}(x)\right|^{q},\left|f^{\prime}(y)\right|^{q}\right)\right\}\right]^{\frac{1}{q}}
\end{gathered}
$$

which completes the desired inequality.

Corollary 1. Set $\eta(x, y)=x-y$ to obtain

$$
\begin{aligned}
& \left|\frac{1}{y-x} f(y)-\frac{\Gamma_{k}(\alpha+k)}{(y-x)^{\frac{\alpha}{k}+1}}{ }_{k} J_{y^{-}}^{\alpha} f(x)\right| \leqslant \\
& \leqslant\left(\frac{k}{\alpha p+k}\right)^{\frac{1}{p}}\left[\max \left\{\left|f^{\prime}(y)\right|^{q},\left|f^{\prime}(x)\right|^{q}\right\}\right]^{\frac{1}{q}} .
\end{aligned}
$$

Corollary 2. Choose $x=a, y=b$ and $\alpha=k=1$ to obtain

$$
\left|f(b)-\frac{1}{b-a} \int_{a}^{b} f(x) d x\right| \leqslant \frac{b-a}{(p+1)^{\frac{1}{p}}}\left[\max \left\{\left|f^{\prime}(b)\right|^{q},\left|f^{\prime}(a)\right|^{q}\right\}\right]^{\frac{1}{q}} .
$$


Lemma 2. Let $f: I \subset \mathbb{R} \rightarrow \mathbb{R}$ be a differentiable function on $I^{\circ}$, where $a, b \in I$ with $t \in[0,1]$. If $f^{\prime} \in L[a, b]$, then we have, for all $a \leqslant x<y \leqslant b$ and $k, \alpha>0$ :

$$
\begin{aligned}
& \frac{1}{x-y}[f(x)-f(y)]+\frac{\Gamma_{k}(\alpha+k)}{(y-x)^{\frac{\alpha}{k}+1}}\left[{ }_{k} J_{x^{+}}^{\alpha} f(y)-{ }_{k} J_{y^{-}}^{\alpha} f(x)\right]= \\
& =\int_{0}^{1} t^{\frac{\alpha}{k}} f^{\prime}(t x+(1-t) y) d t+\int_{0}^{1}(1-t)^{\frac{\alpha}{k}} f^{\prime}(t x+(1-t) y) d t .
\end{aligned}
$$

Proof. Integrating by parts, we can write the integrals above as follows:

$$
\begin{aligned}
& \int_{0}^{1} t^{\frac{\alpha}{k}} f^{\prime}(t x+(1-t) y) d t=\frac{1}{x-y} f(x)+\frac{\Gamma_{k}(\alpha+k)}{(y-x)^{\frac{\alpha}{k}+1}}{ }_{k} J_{x^{+}}^{\alpha} f(y), \\
& \int_{0}^{1}(1-t)^{\frac{\alpha}{k}} f^{\prime}(t x+(1-t) y) d t= \\
&=-\frac{1}{x-y} f(y)-\frac{\frac{\alpha}{k}}{(y-x)^{\frac{\alpha}{k}+1}} \int_{x}^{y}(u-x)^{\frac{\alpha}{k}-1} f(u) d u= \\
&=-\frac{1}{x-y} f(y)-\frac{\Gamma_{k}(\alpha+k)}{(y-x)^{\frac{\alpha}{k}+1}}{ }_{k} J_{y^{-}}^{\alpha} f(x) .
\end{aligned}
$$

Adding the above integral equalities, we get the desired inequality.

Theorem 3. Let $f: I \subset \mathbb{R} \rightarrow \mathbb{R}$ be a differentiable function on $I^{\circ}$, where $a, b \in I$ with $t \in[0,1]$. If $f^{\prime} \in L[a, b]$ and $\left|f^{\prime}\right|$ is a $s$-GL type function, then

$$
\begin{aligned}
& \left|\frac{1}{x-y}[f(x)-f(y)]+\frac{\Gamma_{k}(\alpha+k)}{(y-x)}\left[{ }_{k} J_{x^{+}}^{\alpha} f(y)-{ }_{k} J_{y^{-}}^{\alpha} f(x)\right]\right| \leqslant \\
& \leqslant\left(\left|f^{\prime}(x)\right|+\left|f^{\prime}(y)\right|\right)\left[\beta\left(\frac{\alpha}{k}+1,1-s\right)+\frac{k}{\alpha-k s+k}\right],
\end{aligned}
$$

for all $a \leqslant x<y \leqslant b$ and $k, \alpha>0, s \in[0,1) ; \beta(x, y)=\int_{0}^{1} t^{x-1}(1-t)^{y-1} d t$, $x>1, y>0$ is the Euler Beta function. 
Proof. From Lemma 2 and the properties of the absolute value:

$$
\begin{aligned}
& \left|\int_{0}^{1} t^{\frac{\alpha}{k}} f^{\prime}(t x+(1-t) y) d t+\int_{0}^{1}(1-t)^{\frac{\alpha}{k}} f^{\prime}(t x+(1-t) y) d t\right| \leqslant \\
& \leqslant \int_{0}^{1} t^{\frac{\alpha}{k}}\left|f^{\prime}(t x+(1-t) y)\right| d t+\int_{0}^{1}(1-t)^{\frac{\alpha}{k}}\left|f^{\prime}(t x+(1-t) y)\right| d t .
\end{aligned}
$$

Since $\left|f^{\prime}\right|$ is a $s$-Godunova-Levin type function, we get, applying integration by parts to every integral, respectively:

$$
\begin{aligned}
& \int_{0}^{1} t^{\frac{\alpha}{k}}\left|f^{\prime}(t x+(1-t) y)\right| d t \leqslant\left|f^{\prime}(x)\right| \int_{0}^{1} t^{\frac{\alpha}{k}-s} d t+\left|f^{\prime}(y)\right| \int_{0}^{1} t^{\frac{\alpha}{k}}(1-t)^{-s} d t= \\
& =\left[\frac{k}{\alpha-k s+k}\left|f^{\prime}(x)\right|+\left|f^{\prime}(y)\right| \beta\left(\frac{\alpha}{k}+1,1-s\right)\right]
\end{aligned}
$$

and

$$
\int_{0}^{1}(1-t)^{\frac{\alpha}{k}}\left|f^{\prime}(t x+(1-t) y)\right| d t=\left|f^{\prime}(x)\right| \beta\left(1-s, \frac{\alpha}{k}+1\right)+\frac{k\left|f^{\prime}(y)\right|}{\alpha-k s+k} .
$$

Finally, since $\beta(x, y)=\beta(y, x)$, we have

$$
\begin{gathered}
\left|\frac{1}{x-y}[f(x)-f(y)]+\frac{\Gamma_{k}(\alpha+k)}{(y-x)}\left[{ }_{k} J_{x^{+}}^{\alpha} f(y)-{ }_{k} J_{y^{-}}^{\alpha} f(x)\right]\right| \\
\leqslant\left(\left|f^{\prime}(x)\right|+\left|f^{\prime}(y)\right|\right)\left[\beta\left(\frac{\alpha}{k}+1,1-s\right)+\frac{k}{\alpha-k s+k}\right] .
\end{gathered}
$$

This completes the proof.

Lemma 3. Let $f: I \subset \mathbb{R} \rightarrow \mathbb{R}$ be a twice differentiable function on $I^{\circ}$, where $a, b \in I$ with $t \in[0,1]$. If $f^{\prime \prime} \in L[a, b]$, then for all $a<b$ and $\frac{\alpha}{k}-1>0$, we have

$$
\begin{aligned}
& \frac{k 2^{\frac{\alpha}{k}-2}}{\alpha(b-a)^{\frac{\alpha}{k}-1}} \Gamma_{k}(\alpha+k)\left[{ }_{k} J_{\frac{a+b}{2}}^{\alpha-1} f(b)+{ }_{k} J_{\frac{a+b}{2}}^{\alpha-1} f(a)\right]-f\left(\frac{a+b}{2}\right)= \\
& =\frac{k(b-a)^{2}}{\alpha 2^{2-\frac{\alpha}{k}}}\left[\int_{0}^{\frac{1}{2}} t^{\frac{\alpha}{k}} f^{\prime \prime}(t a+(1-t) b) d t+\int_{\frac{1}{2}}^{1}(1-t)^{\frac{\alpha}{k}} f^{\prime \prime}(t a+(1-t) b) d t\right] .
\end{aligned}
$$


Proof. Integrating by parts several times, we obtain the following equalities:

$$
\begin{gathered}
\int_{0}^{\frac{1}{2}} t^{\frac{\alpha}{k}} f^{\prime \prime}(t a+(1-t) b) d t=\frac{f^{\prime}\left(\frac{a+b}{2}\right)}{(a-b) 2^{\frac{\alpha}{k}}}-\frac{\frac{\alpha}{k}}{(a-b)^{2} 2^{\frac{\alpha}{k}-1}} f\left(\frac{a+b}{2}\right)+ \\
\quad+\frac{\frac{\alpha}{k}\left(\frac{\alpha}{k}-1\right)}{(a-b)^{2}} \int_{0}^{\frac{1}{2}} t^{\frac{\alpha}{k}-2} f(t a+(1-t) b) d t \\
\int_{\frac{1}{2}(1-t)^{\frac{\alpha}{k}} f^{\prime \prime}(t a+(1-t) b) d t=-\frac{f^{\prime}\left(\frac{a+b}{2}\right)}{(a-b) 2^{\frac{\alpha}{k}}}-\frac{\frac{\alpha}{k}}{(a-b)^{2} 2^{\frac{\alpha}{k}-1}} f\left(\frac{a+b}{2}\right)+}^{1}+\frac{\frac{\alpha}{k}\left(\frac{\alpha}{k}-1\right)}{(a-b)^{2}} \int_{\frac{1}{2}}^{1}(1-t)^{\frac{\alpha}{k}-2} f(t a+(1-t) b) d t .
\end{gathered}
$$

Now, changing the variable $u=t a+(1-t) b$ in every integral, we have

$$
\begin{aligned}
\int_{0}^{\frac{1}{2}} t^{\frac{\alpha}{k}-2} f(t a+(1-t) b) d t=\frac{1}{(b-a)^{\frac{\alpha}{k}-1}} \int_{\frac{a+b}{2}}^{b}(b-u)^{\frac{\alpha}{k}-2} f(u) d u= \\
=\frac{k \Gamma\left(\frac{\alpha}{k}-1\right)}{(b-a)^{\frac{\alpha}{k}-1}}{ }_{k} J_{\frac{a+b}{2}}^{\alpha-1} f(b),
\end{aligned}
$$

$$
\begin{array}{r}
\int_{\frac{1}{2}}^{1}(1-t)^{\frac{\alpha}{k}-2} f(t a+(1-t) b) d t=\frac{1}{(b-a)^{\frac{\alpha}{k}-1}} \int_{a}^{\frac{a+b}{2}}(u-a)^{\frac{\alpha}{k}-2} f(u) d u= \\
=\frac{k \Gamma\left(\frac{\alpha}{k}-1\right)}{(b-a)^{\frac{\alpha}{k}-1}}{ }_{k} J_{\frac{a+b}{2}}^{\alpha-1} f(a) .
\end{array}
$$

By adding these inequalities and multiplying by $\frac{\alpha}{k(a-b)^{2} 2^{\frac{\alpha}{k}-1}}$, we get the required inequality. 
Remark 1. Taking $k=1$ in Lemma 3, we obtain equality (2) from [2] for $m=1$.

Theorem 4. Let $f: I \subset \mathbb{R} \rightarrow \mathbb{R}$ be a differentiable function on $I^{\circ}$, such that $f^{\prime \prime} \in L[a, b]$, where $a, b \in I, a<b$ with $t \in[0,1]$. If $\left|f^{\prime \prime}\right|^{q}$ is $\eta$-QC on $[a, b] \subset I$ and $q \geqslant 1$, then the following inequality for fractional integrals holds:

$$
\begin{aligned}
& \left|\frac{k 2^{\frac{\alpha}{k}-2}}{\alpha(b-a)^{\frac{\alpha}{k}-1}} \Gamma_{k}(\alpha+k)\left[J_{k} J_{\frac{a+b}{2}}^{\alpha-1} f(b)+{ }_{k} J_{\frac{a+b}{2}}^{\alpha-1} f(a)\right]-f\left(\frac{a+b}{2}\right)\right| \leqslant \\
& \leqslant\left(\frac{b-a}{2}\right)^{2} \frac{k^{2}}{\alpha(\alpha+k)}\left[\max \left(\left|f^{\prime \prime}(b)\right|^{q},\left|f^{\prime \prime}(b)\right|^{q}+\eta\left(\left|f^{\prime \prime}(a)\right|^{q},\left|f^{\prime \prime}(b)\right|^{q}\right)\right)\right]^{\frac{1}{q}},
\end{aligned}
$$

where $\frac{\alpha}{k}-1>0$.

Proof. Using Lemma 3, the power-mean inequality, and properties of absolute value, we can write

$$
\begin{aligned}
& \left|\frac{k 2^{\frac{\alpha}{k}-2}}{\alpha(b-a)^{\frac{\alpha}{k}-1}} \Gamma_{k}(\alpha+k)\left[{ }_{k} J_{\frac{a+b}{2}}^{\alpha-1} f(b)+{ }_{k} J_{\frac{a+b}{2}}^{\alpha-1} f(a)\right]-f\left(\frac{a+b}{2}\right)\right| \leqslant \\
& \leqslant \frac{k(b-a)^{2}}{\alpha 2^{2-\frac{\alpha}{k}}}\left[\int_{0}^{\frac{1}{2}} t^{\frac{\alpha}{k}}+\int_{\frac{1}{2}}^{1}(1-t)^{\frac{\alpha}{k}}\right]\left|f^{\prime \prime}(t a+(1-t) b)\right| d t \leqslant \\
& \leqslant \frac{k(b-a)^{2}}{\alpha 2^{2-\frac{\alpha}{k}}}\left(\int_{0}^{\frac{1}{2}} t^{\frac{\alpha}{k}} d t\right)^{1-\frac{1}{q}}\left(\int_{0}^{\frac{1}{2}} t^{\frac{\alpha}{k}}\left|f^{\prime \prime}(t a+(1-t) b)\right|^{q} d t\right)^{\frac{1}{q}}+ \\
& +\left(\int_{\frac{1}{2}}^{1}(1-t)^{\frac{\alpha}{k}} d t\right)^{1-\frac{1}{q}}\left(\int_{\frac{1}{2}}^{1}(1-t)^{\frac{\alpha}{k}}\left|f^{\prime \prime}(t a+(1-t) b)\right|^{q} d t\right)^{\frac{1}{q}}= \\
& =\left(\frac{b-a}{2}\right)^{2} \frac{k^{2}}{\alpha(\alpha+k)}\left[\max \left(\left|f^{\prime \prime}(b)\right|^{q},\left|f^{\prime \prime}(b)\right|^{q}+\eta\left(\left|f^{\prime \prime}(a)\right|^{q},\left|f^{\prime \prime}(b)\right|^{q}\right)\right)\right]^{\frac{1}{q}} .
\end{aligned}
$$

And it can be easily checked that $\int_{0}^{\frac{1}{2}} t^{\frac{\alpha}{k}} d t=\int_{\frac{1}{2}}^{1}(1-t)^{\frac{\alpha}{k}} d t=\frac{k}{2^{\frac{\alpha}{k}+1}(\alpha+k)}$ this completes the proof.

Corollary 1. Set $\eta(a, b)=a-b$ in Theorem 4 to obtain 


$$
\begin{gathered}
\left|\frac{k 2^{\frac{\alpha}{k}-2}}{\alpha(b-a)^{\frac{\alpha}{k}-1}} \Gamma_{k}(\alpha+k)\left[{ }_{k} J_{\frac{a+b}{2}}^{\alpha-1} f(b)+{ }_{k} J_{\frac{a+b}{2}}^{\alpha-1} f(a)\right]-f\left(\frac{a+b}{2}\right)\right| \leqslant \\
\leqslant\left(\frac{b-a}{2}\right)^{2} \frac{k^{2}}{\alpha(\alpha+k)}\left[\max \left(\left|f^{\prime \prime}(b)\right|^{q},\left|f^{\prime \prime}(a)\right|^{q}\right)\right]^{\frac{1}{q}} .
\end{gathered}
$$

Corollary 2. Let $a, b \in \mathbb{R}^{+}$and $a<b$. Choose $\alpha=1$ and $k=1$ in (4) to obtain an estimate for Jensen's inequality:

$$
\left|\frac{f(b)+f(a)}{2}-f\left(\frac{a+b}{2}\right)\right| \leqslant \frac{(b-a)^{2}}{8}\left[\max \left(\left|f^{\prime \prime}(b)\right|^{q},\left|f^{\prime \prime}(a)\right|^{q}\right)\right]^{\frac{1}{q}} .
$$

Proof. Indeed, for $\alpha=1$ and $k=1$ we can rewrite the left-hand side of (4):

$$
\begin{array}{r}
\left|\frac{k 2^{\frac{\alpha}{k}-2}}{\alpha(b-a)^{\frac{\alpha}{k}-1}} \Gamma_{k}(\alpha+k)\left[{ }_{k} J_{\frac{a+b}{2}}^{\alpha-1} f(b)+{ }_{k} J_{\frac{a+b}{2}}^{\alpha-1} f(a)\right]-f\left(\frac{a+b}{2}\right)\right|= \\
=\left|\frac{2^{-1}}{1} \Gamma_{1}(2)\left[J_{\frac{a+b}{2}}^{0} f(b)+J_{\frac{a+b}{2}}^{-0}-f(a)\right]-f\left(\frac{a+b}{2}\right)\right|= \\
=\left|\frac{f(b)+f(a)}{2}-f\left(\frac{a+b}{2}\right)\right|
\end{array}
$$

as well as the right-hand side

$$
\begin{aligned}
& \left(\frac{b-a}{2}\right)^{2} \frac{k^{2}}{\alpha(\alpha+k)}\left[\max \left(\left|f^{\prime \prime}(b)\right|^{q},\left|f^{\prime \prime}(a)\right|^{q}\right)\right]^{\frac{1}{q}}= \\
& =\frac{(b-a)^{2}}{8}\left[\max \left(\left|f^{\prime \prime}(b)\right|^{q},\left|f^{\prime \prime}(a)\right|^{q}\right)\right]^{\frac{1}{q}}
\end{aligned}
$$

This inequality is of the same order as the inequality obtained in [4] (see Corollary 2.2).

Similarly, we can prove the following corollary:

Corollary 3. Let $a, b \in \mathbb{R}^{+}$and $a<b$. Choose $\alpha=2$ and $k=1$ in (4) to obtain

$$
\left|\frac{1}{b-a} \int_{a}^{b} f(x) d x-f\left(\frac{a+b}{2}\right)\right| \leqslant \frac{(b-a)^{2}}{24}\left[\max \left(\left|f^{\prime \prime}(b)\right|^{q},\left|f^{\prime \prime}(a)\right|^{q}\right)\right]^{\frac{1}{q}} .
$$

For $q=1$, this inequality is of the same order as the inequality obtained in [15] (see Prposition 1) and in [2] inequality (11). 
Theorem 5. Let $f: I \subset \mathbb{R} \rightarrow \mathbb{R}, I \subset[0, \infty)$, be a differentiable function on $I^{\circ}$, such that $f, g \in L[a, b]$ and $a, b \in I, 0 \leqslant a<b$. $|f|^{p}$ and $|g|^{q}$ are $\eta$-QC on $[a, b]$ for $t \in[0,1], q>1$. Then, for all $x \in[a, b], \frac{\alpha}{k}+1>0$, $\frac{1}{p}+\frac{1}{q}=1$, the following inequality holds:

$$
\begin{aligned}
& \frac{1}{b-a}\left|\int_{a}^{b} f(x) g(x) h(x) d x\right| \leqslant \\
& \quad \leqslant M^{\frac{1}{p}} N^{\frac{1}{q}}\left[\frac{\Gamma_{k}(\alpha+k)}{(b-a)^{\frac{\alpha}{k}+1}}\left[{ }_{k} J_{a^{+}}^{\alpha} f(b)+{ }_{k} J_{b^{-}}^{\alpha} f(a)\right]-\frac{1}{b-a} \int_{a}^{b} f(x) d x\right],
\end{aligned}
$$

where

$$
\begin{aligned}
M & =\max \left\{|f(b)|^{p},|f(b)|^{p}+\eta\left(|f(a)|^{p},|f(b)|^{p}\right)\right\}, \\
N & =\max \left\{|g(b)|^{q},|g(b)|^{q}+\eta\left(|g(a)|^{q},|g(b)|^{q}\right)\right\},
\end{aligned}
$$

$h(t a+(1-t) b)=\left[(1-t)^{\frac{\alpha}{k}}+\left(t^{\frac{\alpha}{k}}-1\right)\right] f(t a+(1-t) b) \geqslant 0$ for all $t \in[0,1]$ and $\frac{\alpha}{k} \in[0,1]$.

Proof. We will use the weighted Hölder inequality:

$$
\begin{aligned}
& \frac{1}{b-a} \int_{a}^{b} f(x) g(x) h(x) d x= \\
&= \int_{0}^{1} f(t a+(1-t) b) g(t a+(1-t) b) h(t a+(1-t) b) d t \\
& \frac{1}{b-a}\left|\int_{a}^{b} f(x) g(x) h(x) d x\right| \leqslant\left(\int_{0}^{1}|f(t a+(1-t) b)|^{p} h(t a+(1-t) b) d t\right)^{\frac{1}{p}} \times \\
& \quad \times\left(\int_{0}^{1}|g(t a+(1-t) b)|^{q} h(t a+(1-t) b) d t\right)^{\frac{1}{q}} \leqslant \\
& \leqslant\left(\int_{0}^{1} h(t a+(1-t) b) d t\right)^{\frac{1}{p}}\left(\int_{0}^{1} h(t a+(1-t) b) d t\right)^{\frac{1}{q}} \times \\
& \quad \times\left[\max \left\{|f(b)|^{p},|f(b)|^{p}+\eta\left(|f(a)|^{p},|f(b)|^{p}\right)\right\}\right]^{\frac{1}{p}} \times
\end{aligned}
$$




$$
\begin{gathered}
\times\left[\max \left\{|g(b)|^{q},|g(b)|^{q}+\eta\left(|g(a)|^{q},|g(b)|^{q}\right)\right\}\right]^{\frac{1}{q}}= \\
=\left(\int_{0}^{1} h(t a+(1-t) b) d t\right)^{\frac{1}{p}+\frac{1}{q}} \times M^{\frac{1}{p}} N^{\frac{1}{q}}= \\
=M^{\frac{1}{p}} N^{\frac{1}{q}}\left(\int_{0}^{1}\left[(1-t)^{\frac{\alpha}{k}}+\left(t^{\frac{\alpha}{k}}-1\right)\right] f(t a+(1-t) b) d t\right)= \\
=M^{\frac{1}{p}} N^{\frac{1}{q}}\left[\frac{\Gamma_{k}(\alpha+k)}{(b-a)^{\frac{\alpha}{k}+1}}\left[{ }_{k} J_{b^{-}}^{\alpha} f(a)+_{k} J_{a^{+}}^{\alpha} f(b)\right]-\frac{1}{b-a} \int_{a}^{b} f(x) d x\right]
\end{gathered}
$$

this completes the proof.

Corollary 1. Set $\eta(a, b)=a-b$ to obtain

$$
\begin{aligned}
& \frac{1}{b-a}\left|\int_{a}^{b} f(x) g(x) h(x) d x\right| \leqslant \\
& \leqslant\left[\frac{\Gamma_{k}(\alpha+k)}{(b-a)^{\frac{\alpha}{k}+1}}\left[{ }_{k} J_{a^{+}}^{\alpha} f(b)+{ }_{k} J_{b^{-}}^{\alpha} f(a)\right]-\frac{1}{b-a} \int_{a}^{b} f(x) d x\right] \times \\
& \quad \times\left[\max \left\{|f(b)|^{p},|f(a)|^{p}\right\}\right]^{\frac{1}{p}}\left[\max \left\{|g(b)|^{q},|g(a)|^{q}\right\}\right]^{\frac{1}{q}} .
\end{aligned}
$$

Further in this article, the following notation is used:

$$
\begin{aligned}
& U=\frac{\frac{\alpha}{k}+1}{b-a}\left[{ }_{k} J_{b^{-}}^{\alpha} f(b)+{ }_{k} J_{b^{-}}^{\alpha} f(a)\right]-\left[{ }_{k} J_{b^{-}}^{\alpha-1} f(b)+{ }_{k} J_{b^{-}}^{\alpha-1} f(a)\right], \\
& V=\left|f^{\prime \prime}(t a+(1-t) b)\right| \leqslant\left[\max \left\{\left|f^{\prime \prime}(b)\right|,\left|f^{\prime \prime}(b)\right|+\eta\left(\left|f^{\prime \prime}(a)\right|,\left|f^{\prime \prime}(b)\right|\right)\right\}\right] . \\
& W=\left|f^{\prime \prime}(t b+(1-t) a)\right| \leqslant\left[\max \left\{\left|f^{\prime \prime}(a)\right|,\left|f^{\prime \prime}(a)\right|+\eta\left(\left|f^{\prime \prime}(b)\right|,\left|f^{\prime \prime}(a)\right|\right)\right\}\right] .
\end{aligned}
$$

Lemma 4. Let $f: I \subset \mathbb{R} \rightarrow \mathbb{R}$ be a twice differentiable mapping on $I^{\circ}$. If $f^{\prime \prime} \in L[a, b], a, b \in I$, then the following equality holds for all $\frac{\alpha}{k}>1$ :

$$
\frac{f(a)+f(b)}{2}-\frac{\Gamma_{k}(\alpha+k)}{2(b-a)^{\frac{\alpha}{k}-1}} U=\frac{(b-a)^{2}}{2}\left(I_{1}+I_{2}\right),
$$

where

$$
I_{1}=\int_{0}^{1}(1-t) t^{\frac{\alpha}{k}} f^{\prime \prime}((1-t) a+t b) d t
$$




$$
I_{2}=\int_{0}^{1}(1-t) t^{\frac{\alpha}{k}} f^{\prime \prime}((t a+(1-t) b) d t .
$$

Proof. Integrating by parts twice, transform the first integral:

$$
\begin{aligned}
& I_{1}=\int_{0}^{1}(1-t) t^{\frac{\alpha}{k}} f^{\prime \prime}((1-t) a+t b) d t \\
&=\frac{f(b)}{(b-a)^{2}}+\frac{\frac{\alpha}{k}\left(\frac{\alpha}{k}-1\right)}{(b-a)^{2}} \int_{0}^{1} t^{\frac{\alpha}{k}-2} f((1-t) a+t b) d t- \\
& \quad-\frac{\frac{\alpha}{k}\left(\frac{\alpha}{k}+1\right)}{(b-a)^{2}} \int_{0}^{1} t^{\frac{\alpha}{k}-1} f((1-t) a+t b) d t .
\end{aligned}
$$

Make the $(1-t) a+t b=x$ transformation in both obtained integrals and add them, then write:

$$
\begin{aligned}
I_{1}=\frac{f(b)}{(b-a)^{2}}+\frac{\frac{\alpha}{k}\left(\frac{\alpha}{k}-1\right)}{(b-a)^{\frac{\alpha}{k}+1}} \int_{a}^{b}(x-a)^{\frac{\alpha}{k}-2} f(x) d x- & \\
& \quad-\frac{\frac{\alpha}{k}\left(\frac{\alpha}{k}+1\right)}{(b-a)^{\frac{\alpha}{k}+2}} \int_{a}^{b}(x-a)^{\frac{\alpha}{k}-1} f(x) d x
\end{aligned}
$$

or

$$
I_{1}=\frac{f(b)}{(b-a)^{2}}+\frac{\Gamma_{k}(\alpha+k)}{(b-a)^{\frac{\alpha}{k}+1}}{ }_{k} J_{b^{-}}^{\alpha-1} f(a)-\frac{\left(\frac{\alpha}{k}+1\right) \Gamma_{k}(\alpha+k)}{(b-a)^{\frac{\alpha}{k}+2}}{ }_{k} J_{b^{-}}^{\alpha} f(a) .
$$

Similarly, for the other integral

$$
I_{2}=\frac{f(a)}{(b-a)^{2}}+\frac{\Gamma_{k}(\alpha+k)}{(b-a)^{\frac{\alpha}{k}+1}}{ }_{k} J_{b^{-}}^{\alpha-1} f(b)-\frac{\left(\frac{\alpha}{k}+1\right) \Gamma_{k}(\alpha+k)}{(b-a)^{\frac{\alpha}{k}+2}}{ }_{k} J_{b^{-}}^{\alpha} f(b) .
$$

Get, by summing these equalites and then grouping the summands:

$$
\begin{aligned}
I_{1}+I_{2}=\frac{[f(a)+f(b)]}{(b-a)^{2}}- & \frac{\left(\frac{\alpha}{k}+1\right) \Gamma_{k}(\alpha+k)}{(b-a)^{\frac{\alpha}{k}+2}}\left[{ }_{k} J_{b^{-}}^{\alpha} f(b)+{ }_{k} J_{b^{-}}^{\alpha} f(a)\right]+ \\
& +\frac{\Gamma_{k}(\alpha+k)}{(b-a)^{\frac{\alpha}{k}+1}}\left[{ }_{k} J_{b^{-}}^{\alpha-1} f(b)+{ }_{k} J_{b^{-}}^{\alpha-1} f(a)\right] .
\end{aligned}
$$


Obtain (5) by multiplying both sides of equality (6) by the expression $\frac{(b-a)^{2}}{2}$. The proof is completed.

Remark 2. For $k=1$ in Lemma 4, we obtain equality (20) in [2].

Theorem 6. Let $f: I \subset \mathbb{R} \rightarrow \mathbb{R}, I \subset[0, \infty)$, be a differentiable function on $I^{\circ}$, such that $f^{\prime \prime} \in L[a, b]$, where $a, b \in I, a \leqslant x<y \leqslant b$. If $\left|f^{\prime \prime}\right|$ is $\eta$-QC on $[x, y]$ for $t \in[0,1]$, then we have, for all $k, \alpha>0$ :

$$
\left|\frac{f(a)+f(b)}{2}-\frac{\Gamma_{k}(\alpha+k)}{2(b-a)^{\frac{\alpha}{k}-1}} \cdot U\right| \leqslant \frac{(b-a)^{2}}{2\left(\frac{\alpha}{k}+1\right)\left(\frac{\alpha}{k}+2\right)} \times[V+W] .
$$

Proof. Using properties of the absolute value and Lemma 4, we obtain

$$
\begin{aligned}
& \left|\frac{f(a)+f(b)}{2}-\frac{\Gamma_{k}(\alpha+k)}{2(b-a)^{\frac{\alpha}{k}-1}} \cdot U\right| \leqslant \\
& \leqslant \frac{(b-a)^{2}}{2} \int_{0}^{1}(1-t) t^{\frac{\alpha}{k}}\left[\left|f^{\prime \prime}((1-t) a+t b)\right|+\left|f^{\prime \prime}(t a+(1-t) b)\right|\right] d t \leqslant \\
& \leqslant \frac{(b-a)^{2}}{2\left(\frac{\alpha}{k}+1\right)\left(\frac{\alpha}{k}+2\right)}\left[\max \left\{\left|f^{\prime \prime}(b)\right|,\left|f^{\prime \prime}(b)\right|+\eta\left(\left|f^{\prime \prime}(a)\right|,\left|f^{\prime \prime}(b)\right|\right)\right\}\right]+ \\
& +\frac{(b-a)^{2}}{2\left(\frac{\alpha}{k}+1\right)\left(\frac{\alpha}{k}+2\right)}\left[\max \left\{\left|f^{\prime \prime}(a)\right|,\left|f^{\prime \prime}(a)\right|+\eta\left(\left|f^{\prime \prime}(b)\right|,\left|f^{\prime \prime}(a)\right|\right)\right\}\right] .
\end{aligned}
$$

The proof is completed.

Corollary 1. Set $\eta(a, b)=a-b$ in Theorem 6 to obtain

$$
\left|\frac{f(a)+f(b)}{2}-\frac{\Gamma_{k}(\alpha+k)}{2(b-a)^{\frac{\alpha}{k}-1}} \cdot U\right| \leqslant \frac{(b-a)^{2} \max \left\{\left|f^{\prime \prime}(b)\right|,\left|f^{\prime \prime}(a)\right|\right\}}{\left(\frac{\alpha}{k}+1\right)\left(\frac{\alpha}{k}+2\right)} .
$$

\section{New Hölder and improved-iscan inequalities.}

Theorem 7. Let $f: I \subset \mathbb{R} \rightarrow \mathbb{R}, I \subset[0, \infty)$, be a differentiable function on $I^{\circ}$, such that $f^{\prime \prime} \in L[a, b]$, where $a, b \in I, a \leqslant x<y \leqslant b$. If $\left|f^{\prime \prime}\right|^{q}$ is $\eta$-QC on $[x, y]$ for $t \in[0,1], q>1, p=\frac{q}{q-1}$, then we have, for all $k, \alpha>0$ :

$$
\begin{aligned}
& \left|\frac{f(a)+f(b)}{2}-\frac{\Gamma_{k}(\alpha+k)}{2(b-a)^{\frac{\alpha}{k}-1}} \cdot U\right| \leqslant \\
& \leqslant \frac{(b-a)^{2}}{2^{1+\frac{1}{q}}}\left[\left(\beta\left(3, \frac{\alpha}{k} p+1\right)\right)^{\frac{1}{p}}+\left(\beta\left(2, \frac{\alpha}{k} p+2\right)\right)^{\frac{1}{p}}\right]\left(W^{\frac{1}{q}}+V^{\frac{1}{q}}\right) .
\end{aligned}
$$


Proof. Using the Hölder-Iscan inequality (see [10]), properties of the absolute value, and Lemma 4, we obtain

$$
\begin{aligned}
& \left|\frac{f(a)+f(b)}{2}-\frac{\Gamma_{k}(\alpha+k)}{2(b-a)^{\frac{\alpha}{k}-1}} \cdot U\right|= \\
& =\frac{(b-a)^{2}}{2}\left|\int_{0}^{1}(1-t) t^{\frac{\alpha}{k}}\left[f^{\prime \prime}((1-t) a+t b)+f^{\prime \prime}(t a+(1-t) b)\right] d t\right| \leqslant \\
& \leqslant \frac{(b-a)^{2}}{2}\left[\left\{\left(\int_{0}^{1}(1-t)^{2} t^{\frac{\alpha}{k} p} d t\right)^{\frac{1}{p}}\left(\int_{0}^{1}(1-t)\left|f^{\prime \prime}((1-t) a+t b)\right|^{q} d t\right)^{\frac{1}{q}}+\right.\right. \\
& \left.+\left(\int_{0}^{1}(1-t) t^{\frac{\alpha}{k} p+1} d t\right)^{\frac{1}{p}}\left(\int_{0}^{1} t\left|f^{\prime \prime}((1-t) a+t b)\right|^{q} d t\right)^{\frac{1}{q}}\right\}+ \\
& +\left\{\left(\int_{0}^{1}(1-t)^{2} t^{\frac{\alpha}{k} p} d t\right)^{\frac{1}{p}}\left(\int_{0}^{1}(1-t)\left|f^{\prime \prime}((1-t) b+t a)\right|^{q} d t\right)^{\frac{1}{q}}+\right. \\
& \left.\left.+\left(\int_{0}^{1}(1-t) t^{\frac{\alpha}{k} p+1} d t\right)^{\frac{1}{p}}\left(\int_{0}^{1} t\left|f^{\prime \prime}((1-t) b+t a)\right|^{q} d t\right)^{\frac{1}{q}}\right\}\right] .
\end{aligned}
$$

Given the fact that

$$
\int_{0}^{1}(1-t)^{2} t^{\frac{\alpha}{k} p} d t=\beta\left(3, \frac{\alpha}{k} p+1\right) \text { and } \int_{0}^{1}(1-t) t^{\frac{\alpha}{k} p+1} d t=\beta\left(2, \frac{\alpha}{k} p+2\right),
$$

we can write

$$
\begin{aligned}
& \left|\frac{f(a)+f(b)}{2}-\frac{\Gamma_{k}(\alpha+k)}{2(b-a)^{\frac{\alpha}{k}-1}} \cdot U\right| \leqslant \\
& \quad \leqslant \frac{(b-a)^{2}}{2^{1+\frac{1}{q}}}\left[\left(\beta\left(3, \frac{\alpha}{k} p+1\right)\right)^{\frac{1}{p}}+\left(\beta\left(2, \frac{\alpha}{k} p+2\right)\right)^{\frac{1}{p}}\right]\left(W^{\frac{1}{q}}+V^{\frac{1}{q}}\right) .
\end{aligned}
$$

The proof is completed.

Theorem 8. Let $f: I \subset \mathbb{R} \rightarrow \mathbb{R}, I \subset[0, \infty)$, be a differentiable function on $I^{\circ}$, such that $f^{\prime \prime} \in L[a, b]$, where $a, b \in I, a \leqslant x<y \leqslant b$. If $\left|f^{\prime \prime}\right|^{q}$ is $\eta$-QC on $[x, y]$ for $t \in[0,1], q>1, p=\frac{q}{q-1}$, then we have, for all $k, \alpha>0$ :

$$
\left|\frac{f(a)+f(b)}{2}-\frac{\Gamma_{k}(\alpha+k)}{2(b-a)^{\frac{\alpha}{k}-1}} \cdot U\right| \leqslant \frac{(b-a)^{2}}{2} \cdot S \cdot\left(W^{\frac{1}{q}}+V^{\frac{1}{q}}\right),
$$


where $S=\frac{k^{2}}{(\alpha+k)(\alpha+2 k)}$.

Proof. Using the improved power-mean integral inequality, properties of the absolute value, and Lemma 4, we obtain

$$
\begin{aligned}
& \left|\frac{f(a)+f(b)}{2}-\frac{\Gamma_{k}(\alpha+k)}{2(b-a)^{\frac{\alpha}{k}-1}} \cdot U\right| \leqslant \\
& \leqslant \frac{(b-a)^{2}}{2}\left[\left\{\left(\int_{0}^{1}(1-t)^{2} t^{\frac{\alpha}{k}} d t\right)^{1-\frac{1}{q}}\left(\int_{0}^{1}(1-t)^{2} t^{\frac{\alpha}{k}}\left|f^{\prime \prime}((1-t) a+t b)\right|^{q} d t\right)^{\frac{1}{q}}+\right.\right. \\
& \left.+\left(\int_{0}^{1}(1-t) t^{\frac{\alpha}{k}+1} d t\right)^{1-\frac{1}{q}}\left(\int_{0}^{1}(1-t) t^{\frac{\alpha}{k}+1}\left|f^{\prime \prime}((1-t) a+t b)\right|^{q} d t\right)^{\frac{1}{q}}\right\}+ \\
& \quad+\left\{\left(\int_{0}^{1}(1-t)^{2} t^{\frac{\alpha}{k}} d t\right)^{1-\frac{1}{q}}\left(\int_{0}^{1}(1-t)^{2} t^{\frac{\alpha}{k}}\left|f^{\prime \prime}((1-t) b+t a)\right|^{q} d t\right)^{\frac{1}{q}}+\right. \\
& \left.\left.\quad+\left(\int_{0}^{1}(1-t) t^{\frac{\alpha}{k}+1} d t\right)^{1-\frac{1}{q}}\left(\int_{0}^{1}(1-t) t^{\frac{\alpha}{k}+1}\left|f^{\prime \prime}((1-t) b+t a)\right|^{q} d t\right)^{\frac{1}{q}}\right\}\right]
\end{aligned}
$$

Or

$$
\begin{aligned}
& \left|\frac{f(a)+f(b)}{2}-\frac{\Gamma_{k}(\alpha+k)}{2(b-a)^{\frac{\alpha}{k}-1}} \cdot U\right| \leqslant \\
& \leqslant \frac{(b-a)^{2}}{2}\left[\int_{0}^{1}(1-t)^{2} t^{\frac{\alpha}{k}} d t+\int_{0}^{1}(1-t) t^{\frac{\alpha}{k}+1} d t\right]\left(W^{\frac{1}{q}}+V^{\frac{1}{q}}\right) \leqslant \\
& \leqslant \frac{(b-a)^{2}\left(W^{\frac{1}{q}}+V^{\frac{1}{q}}\right)}{2} \int_{0}^{1}(1-t) t^{\frac{\alpha}{k}}[(1-t)+t] d t \leqslant \\
& \leqslant \frac{(b-a)^{2}}{2} \frac{k^{2}}{(\alpha+k)(\alpha+2 k)}\left(W^{\frac{1}{q}}+V^{\frac{1}{q}}\right) .
\end{aligned}
$$

The proof is completed.

4. Applications to special means. Now we consider some means for arbitrary real numbers $\alpha, \beta$.

1) Arithmetic mean : $A(\alpha, \beta)=\frac{\alpha+\beta}{2}$, 
2) Geometric mean: $G(\alpha, \beta)=\sqrt{\alpha \beta}, \alpha \beta \geqslant 0$

3) Logarithmic mean: $L(\alpha, \beta)=\frac{\alpha-\beta}{\ln |\alpha|-\ln |\beta|},|\alpha| \neq|\beta|, \alpha, \beta \neq 0$,

4) Generalized log-mean: $L_{n}(\alpha, \beta)=\left[\frac{\beta^{n+1}-\alpha^{n+1}}{(n+1)(\beta-\alpha)}\right]^{\frac{1}{n}}, n \in \mathbb{Z} \backslash\{-1,0\}$, $\alpha, \beta>0$.

5) The Identric Mean: $I(\alpha, \beta)=\frac{1}{e}\left[\frac{\beta^{\beta}}{\alpha^{\alpha}}\right]^{\frac{1}{\beta-\alpha}}, \alpha \neq \beta$ and $\alpha, \beta>0$.

Now, using the obtained results, we give some applications to special means of real numbers.

Proposition 1. Let $a, b \in \mathbb{R}^{+}$and $a<b$; then we have

$$
I(a, b) \leqslant b e^{\frac{a-b}{2 b}} .
$$

Proof. The assertion follows from Corollary 2 applied to the QC mapping $\alpha=k=1$ and $f(x)=-\ln (x)$, where $x \in \mathbb{R}^{+}$.

Proposition 2. Let $a, b \in \mathbb{R}^{+}, a<b$. Then we have, for all $q \geqslant 1$ :

$$
\left|\ln \frac{I(a, b)}{b}\right| \leqslant \frac{b-a}{2 a} .
$$

Proof. The assertion follows from Corollary 2 applied to the QC mapping $\alpha=k=1$ and $f(x)=-\ln (x)$, where $x \in \mathbb{R}^{+}$.

Remark 3. Obviously, the upper bound (8) for $f(x)=-\ln (x)$ is better than (9).

Proposition 3. Let $a, b \in[e, \infty)$ and $a<b$. Then we have

$$
\left|\frac{A(b \ln a, a \ln b)}{G^{2}(a, b)}-\frac{A(\ln a, \ln b)}{L(b, a)}\right| \leqslant \frac{(b-a)^{2}}{12} \cdot \frac{2 \ln b-3}{b^{3}} .
$$

Proof. The assertion follows from (7) applied to the QC mapping $f(x)=\frac{\ln x}{x}, x \in[e, \infty), \alpha=2$ and $k=1$ (indeed, since the function $f(x)$ is on the interval $[e, \infty)$ decreases, it is $\mathrm{QC})$.

Proposition 4. Let $a, b \in\left[\frac{1}{e}, \infty\right)$ and $a<b$. Then we have

$$
\left|A(a \ln a, b \ln b)-\left[A(a, b) \ln b+\frac{a^{2}}{2} L^{-1}(b, a)-\frac{1}{2} A(a, b)\right]\right| \leqslant \frac{(b-a)^{2}}{12 a} .
$$

Proof. The assertion follows from (7) applied to the QC mapping $f(x)=x \ln x, x \in\left[\frac{1}{e}, \infty\right), \alpha=2$ and $k=1$ (indeed, since the function $f(x)$ is on the interval $[e, \infty)$ and decreases, it is $\mathrm{QC})$. 


\section{References}

[1] Bayraktar B. Some New Inequalities of Hermite-Hadamard Type for Differentiable Godunova-Levin Functions via Fractional Integrals. Konuralp Journal of Mathematics., 2020, vol. 8(1), pp. 91-96.

https://dergipark.org.tr/en/download/article-file/1069475

[2] Bayraktar B. Some Integral Inequalities of Hermite-Hadamard Type for Differentable $(s, m)$-Convex Functions Via Fractional Integrals. TWMS J. App. Eng. Math., 2020, vol. 10(3), pp. 625-637.

https://www.researchgate.net/publication/331894522

[3] Bayraktar B. Some New Generalizations Of Hadamard-Type Midpoint Inequalities Involving Fractional Integrals. Problemy Analiza - Issues of Analysis., 2020, vol. $9(27-3)$, pp. $66-82$.

DOI: http://doi.org/10.15393/j3.art.2020.8270

[4] Bayraktar B., and Gürbüz M. On Some Integral Inequalities for (s, m)-Convex Functions. TWMS J. App. Eng. Math., 2020, vol. 10(2), pp. 288 - 295. https://www. researchgate. net/publication/329686772

[5] Butt S. I., Kashuri A., Umar M., Aslam A., and Gao W. Hermite-JensenMercer type inequalities via $\Psi$-Riemann-Liouville $k$-fractional integrals. AIMS Mathematics., 2020, vol. 5(5), pp. 5193-5220.

DOI: http://doi.org/10.3934/math. 2020334

[6] Butt S. I., Akdemir A. O., Bhatti M. Y., and Nadeem M. New refinements of Chebyshev-Pólya-Szegö-type inequalities via generalized fractional integral operators. Journal of Inequalities and Applications., 2020, Art. No: 157. DOI: http://dx.doi.org/10.1186/s13660-020-02425-6

[7] Diaz R., and Pariguan E. On hypergeometric functions and Pochhammer k-symbol. Divulgaciones Matematicas., 2007, vol. 15(2), pp. 179-192. https://arxiv.org/pdf/math/0405596.pdf

[8] Dragomir S. S. Ineuqalities of Hermite-Hadamard type for h-convex functions on linear spaces. RGMIA., 2013, vol. 16, Art. No: 72.

DOI: http://dx.doi.org/10.4067/S0716-09172015000400002

[9] Gordji M. E., Delavar M. R., and Sen M. D. L. On $\phi$-Convex Functions, Journal of Mathematical Inequalities., 2016, vol. 10(1), pp. 173-183.

DOI: https://doi.org/10.7153/jmi-10-15

[10] Işcan I. New refinements for integral and sum forms of Holder inequality. Journal of Inequalities and Applications., 2019, Art. No: 30.

DOI: https://doi.org/10.1186/s13660-019-2258-5

[11] Nisar K. S., Rahman G., Choi J., Mubeen S., and Arshad M. Certain Gronwall type inequalities associated Riemann-Liouville $k$ - and Hadamard 
$k$-fractional derivatives and their applications. East Asian Math. j., 2018, vol. 34(3), pp. 249-263. DOI: https://doi.org/10.7858/eamj .2018.018

[12] Qaisar S., Iqbal M., Hussain S., Butt S. I., and Meraj M. A. New In-

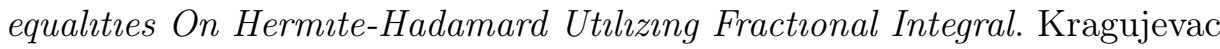
Journal of Mathematics., 2018, vol. 42(1), pp. 15-27.

DOI: https://doi.org/10.5937/KgJMath1801015Q

[13] Qi F., Habib S., Mubeen S., and Naeem M. N. Generalized k-fractional conformable integral and related inequalities. AIMS Mathematics., 2019, vol. 4, pp. 343-358. DOI: https://doi.org/10.3934/math.2019.3.343

[14] Mubeen S. and Habibullah G. M. $k$-Fractional Integrals and Application. Int. J. Contemp. Math. Sciences, 2012, vol. 7(2), pp. 89-94.

[15] Sarıkaya M. Z. and Aktan N. On the generalization of some integral inequalities and their applications. Math. Comput. Modelling., 2011, vol. 54, pp. 2175-2182. DOI: https://doi.org/10.1016/j.mcm.2011.05.026

[16] Set E., Tomar M. and Sarikaya M. Z. On generalized Gruss type inequalities via $k$-Riemann-Liouville fractional integral. Appl. Math. Comput., 2015, vol. 269, pp. 77-89. DOI: https://doi.org/10.1016/j.amc.2015.07.026

[17] Zhao S., Butt S. I., Nazeer W., Nasir J., Umar M., and Liu Y. Some Hermite-Jensen-Mercer type inequalities for $k$-Caputo-fractional derivatives and related results. Advances in Difference Equations., 2020, Art. No:262. DOI: http://doi.org/10.1186/s13662-020-02693-y

Received July 22, 2020.

In revised form, November 30, 2020.

Accepted December 11, 2020.

Published online January 6, 2021.

Saad Ihsan Butt ${ }^{a}$, Bahtiyar Bayraktar ${ }^{b}$, and Muhammad Umar ${ }^{a}$

a. COMSATS University Islamabad, Lahore Campus,

Defence Road Off Raiwind Rd, Lda Avenue Phase 1 Lda Avenue,

Lahore, Punjab 54000, Pakistan

E-mail: saadihsanbutt@gmail.com., umarqureshi987@gmail.com.

b. Bursa ULUDAĞ UNIVERSITY, Faculty of Education, Department of Mathematics and Science Education,

Görukle Campus, 16059, BURSA, TURKEY

E-mail: bbayraktar@uludag.edu.tr. 